\section{Structure and Motion in Polymer Glasses}

\author{
H.H. Kausch, Lausanne
}

Under the title that heads this report, the 10th Europhysics Conference on Macromolecular Physics was held in Noordwijkerhout (the Netherlands) from April 21-25, 1980. About 120 participants from (12) western and (4) eastern European countries, the US and Canada came together in the Leeuwenhorst Congress Centre, splendidly located, all alone in the middle of square miles of colourful tulip fields. The organizing comittee, headed by Dr. J. Heijboer of TNO, Delft, had set up an excellent fourday programme which must be commended for its balance between invited lectures (16), contributed papers (19), posters (16), general discussion sessions (1), excursions (to the overwhelmingly beautiful Keukenhof) and time for discussions and social get-togethers (10 min after each paper, 30 to $45 \mathrm{~min}$ at the end of each session). In order that the theme of the conference structure and mobility - be not too pretentious and intractable, the organizers had concentrated on the amorphous solid state $\left(T<T_{g}\right)$ and on a discussion of the various experimental methods. They succeeded in offering an almost complete coverage.

From the "points of departure" (Heijboer, Delft; McCrum, Oxford) and the classical study of dielectric relaxation (Williams, Aberystwyth), the programme spanned structure and structural relaxation (Wendorff, Darmstadt; Kovacs, Strasbourg; Haward, Birmingham; Hedvig, Budapest; Koppelmann, Leoben; Kolarik, Prague), and went on to dynamic (Read, Teddington), thermal (Lacabanne, Toulouse), electric (Kryszewski, Lodz; Klason, Gothenburg), fluorescence (Monnerie, Paris), and spectroscopic experimental techniques (Boyer, Midland; Veeman, Nijmegen). The conference preprints (Europhysics Conference Abstracts, Vol. 4A) are particularly valuable and make a really useful addition to the literature. They not only give more space to extended abstracts than previously, but contain fulllength introductory papers and an excellent bibliography, covering the latest developments in this field.

According to the number of presentations, the temperature region attracting the most attention is that between the $\alpha$ - and $\beta$-relaxation peaks. These peaks correspond respectively to the reorganisation of the whole structure $(\alpha)$ and to localized motions of side-groups or main-chain segments $(\beta)$. Furthermore, the general discussion session which this writer had the privilege to conduct, centred around the nature and kinetics of creep was also in this temperature range. Struik (Delft) in his introduction had raised the question of the origin of the generality of the creep law: compliance $J(t)=J_{o} \exp +\left(t / t_{o}\right)^{n}$ for a wide selection of different materials. An animated discussion led to the conclusion that the initial phase of (transient) creep must be considered as a statistical phenomenon, associated with the drive towards a new, stress-dependent state of equilibrium of the random and defect-loaded aggregate of (anisotropic) small particles or chain segments. The chain-like nature and thus many polymer characteristics, do not enter the exponent $n$ which is found to vary closely around $1 / 3$.

In most (although surprisingly enough not in all) presentations, the nature of the molecular motion was discussed. But even when this was done, there seemed to be a certain lack of cross-information, indicating that serious thought had not been given to considering how a specified molecular motion couples with different modes of external excitation. This fact underlines the necessity for conferences of this type, and the desirability of using different methods to look at the same material. Although this is best done within one laboratory or by exchange of samples, the participants of this meeting profitted from the fact that the European efforts concentrate somewhat on polyacrylates (e.g. PEA, PMMA, PEMA), polycarbonate (PC), polyvinylchloride (PVC), and polyester (e.g. PETB).

\section{Workshop on Solar Radio Noise Storms}

We report here on a very productive workshop that was held under the auspices of EPS and with the support of CNRS, to consider an astrophysical subject of topical interest. It took place in the 12th century Abbaye de Sénanque (Provence, France) on 17-21 March of this year. A total of 32 invited participants from 10 different European countries (plus USA and Australia) attended. only two thirds of the participants were specialists in the field and were there mainly as observers. Moreover, the rest were not as might have been expected, people from fields closely related to solar radio physics, but from fields of research, fundamental to the problem of noise storms, such as plasma physics and solar MHD, and fields concerned with similar physics, such as planetary radio and magnetospheric physics.
Contrary to the more normal practice,
Apart from focussing on $\alpha$-, $\beta$-, and $\gamma$ relaxation mechanisms, the conference also dealt with the cryogenic temperature range and such fascinating observations as the anomaly in longitudinal sound velocity and dielectric constant in PMMA below $5 \mathrm{~K}$ ! (Hunklinger, Stuttgart), the fracture of polyethylene after plastic deformation at a strain $4.5 \%$ to $10 \%$ at 4.2 to $77 \mathrm{~K}$ (Hartwig, Karlsruhe), the deformation instability in cold drawing of PETP leading to a macrocrazed structure (Pakula, Mainz and Lodz), the detection of minute quantities of crystallinity by light scattering at $190^{\circ} \mathrm{C}$, in otherwise amorphous PC (Dettenmaier, Lausanne), and the mapping of twodimensional $\mathrm{X}$-ray patterns indicating a bent conformation of PMMA segments (Windle, Cambridge).

One may say that there are two current centres of interest. One concerns a quantitative mathematical-physical description of the observed changes as a function of temperature and time. The other concerns the morphological interpretation of relaxation and deformation mechanisms. A thorough understanding leading to valid predictions of polymer behaviour requires, of course, that both aspects be considered simultaneously.

Reviewing the general impression obtained from this conference, one may say that in order to have a good conference, one needs a stimulating subject, an experienced organizing committee, interested and interesting participants, and an attractive location with the weather a little bit on the cool and rainy side, since one would like to have the participants in the auditorium and not in the tulip fields. All this was fulfilled in Leeuwenhorst.

\section{A.O. Benz and}

\section{J.L. Bougeret, Meudon}

The workshop had the goal to start new work in the field of noise storms, and after two days of introductory reviews, the participants split into working groups. At the end, we concluded that our unusual approach had been very successful.

What did we achieve? We learned to ask the right questions, for example: What drives this phenomenon of solar noise storms? the answer to which seems to be the dissipation of emerging magnetic flux from the solar surface (photosphere). The driver may be a current, associated with a shock wave, that is perpendicular to the magnetic field. The ideas generated have led to several proposals for new observations which will be carried out during the current phase of solar activity.

The Proceedings of the workshop under the title, Solarnoistorm, are available from Dr. Jean-Louis Bougeret, Observatoire de Paris, F-92190 Meudon. 